\section{Making waves on Shelter Island}

\author{
Ya. B. Zeldovich
}

Shelter Island II. Proceedings of the $\mathbf{1 9 8 3}$ Shelter Island Conference on Quantum Field Theory and the Fundamental Problems of Physics. Edited by Roman Jackiw, Nicola N. Khuri, Steven Weinberg and Edward Witten. MIT Press: 1985. Pp. 369. $\$ 37.50, £ 37.50$.

THE proceedings contained in this book are best viewed against the background of the evolution of physics in the twentieth century and, more specifically, against events at the previous Shelter Island conference of 1947. That meeting was organized by Robert Oppenheimer and was attended by many talented scientists who had returned to fundamental research after working on war projects.

The intermittent character of scientific progress is now well-recognized; bursts of fermentation of new ideas are punctuated by steady elucidation of all the consequences. A Russian poet Tjutchev, practically unknown abroad, said "Blessed are those who come into this world at its fateful moments"; for physicists the remark can be paraphrased as "Lucky are those who attend conferences when fresh ideas are in the air".

One such period was between 1905 and 1928 , which witnessed the advent of special and general relativity, quantum mechanics, the quantum theory of fields and the theory of antiparticles. In the 1930s, development slowed down: the refinement of theory was plagued by infinities. A theory of nuclear forces was proposed, with heavy particles - mesons being an essential part of it. Particles with approximately the appropriate mass were found in cosmic rays, but the euphoria turned to desperation when they were found not to fit the theory. Many eminent physicists believed that a new conceptual framework would be necessary, something as revolutionary as relativity and quantum theory: as Bohr is reputed to have said: "This theory is not crazy enough to be true".

But the younger generation of the time (and many of the old) were more sober. Shelter Island I gave impetus to the work of Schwinger, Feynman and Bethe, whose renormalization programme brilliantly explained the Lamb-Rutherford level shift in the hydrogen atom and the anomalous magnetic moment of the electron. Marshak went on to consider the possibility of two mesons: the muon as a heavy electron and the pion giving nuclear forces. For several decades physics was dominated by ingenious applications of principles formulated before 1930; even gauge theory and quarks fall in this category. It was not until the mid-1970s and 1980 s that a further wave of ideas arose, and it is these new ideas that are the subject of this volume.

In a nutshell, the goal of the old theory was this: given the existence and characteristics of particles and fields, how could one move on to calculate all processes, bound states and so on? The new theory has a more ambitious programme. It aims at explaining why all known particles exist - and determining their masses and charges. The same theory also predicts the existence of many as yet unknown particles and fields. Very strange - even "crazy" - ideas are needed!

An excellent overall view of how things stand is given in the first paper in the book, where Murray Gell-Mann provides a coherent yet witty account of how theory has developed between the two Shelter Island conferences. Here, too, Gell-Mann summarizes the topics treated in detail in the following articles - supersymmetry, Kaluza-Klein multidimensional theories, superstrings, and the new and fruitful symbiosis between particle physics and astrophysics.

To a reader not well acquainted with particle physics, I should also add mention of two events of previous history: the introduction of Planck units of mass, length and time in 1899 , and the appearance of Dirac's famous paper on large numbers in physics: the ratio of electrostatic to gravitational interaction, $10^{39}$ in the atom, for example. The modern answer is quite different from that proposed by Dirac, but that does not invalidate the importance of the question he raised.

Characteristic of the new wave of ideas is Steven Weinberg's paper, "Calculation of Fine Structure Constants", in which he discusses the now popular idea of higher dimensional space-time manifolds. Time and three spatial coordinates describe the usual space-time, while the extra spatial coordinates are assumed to be "compactified" to dimensions of the order of $10^{-32} \mathrm{~cm}$. They are not observable as coordinates, but their deformations manifest themselves as fields and particles. As shown in Witten's contribution later in the book, the structure of these extra dimensions may explain the spectrum of fermions - the existence of three, and only three, families of leptons and quarks.

This is a revival of a theory, initiated in the 1920 s by Kaluza and Klein, to unify gravity and electromagnetism. For a long time Einstein's own search for a unified theory appeared to be simplistic and to be contradicted by the proliferation of fields and particles found by experimenters. Now, however, most theorists are convinced that the time is ripe for brainstorming in this direction but with more dimensions and a peculiar symmetry (supersymmetry) between bosons and fermions.

A new branch of physics, as compared with the 1947 conference, is the interdisciplinary study of the very early Universe. Then, because of the negative pressure of a scalar field, one had gravitational repulsion instead of attraction and the radius of the Universe grew exponentially. Both Guth and Linde discuss the topic. (Incidentally, Frenkel and Landau from the USSR were invited to attend Shelter Island I, but neither of them went; Linde, of the Lebedev Institute in Moscow, did attend in 1983, and contributed. The ratio $1: 0$ is a great improvement!) In the $1960 \mathrm{~s}$, the big bang theory of the origin of the Universe was supported by the discovery of background microwave radiation in the Universe, and by the theory of nucleosynthesis occurring at temperatures from $3 \times$ $10^{10}$ to $3 \times 10^{8} \mathrm{~K}$. These energies are within the range of our particle accelerators, results from which have lent further weight to the big bang theory. But in attempting to go back to an even earlier stage one enters unexplored territory, and the Universe itself has to play the role of the poor man's accelerator. Here the astrophysicist's work is akin to that of the palaeontologist, in which events in a past era must be interpreted from their traces observed today. For example, in my opinion (shared by many others, including GellMann) the very charge asymmetry of the Universe, that is, the absence of bulk antimatter, is proof of the instability of the proton, which Earth-bound experimentalists have searched for in vain.

Everybody working on or interested in the fundamental problems of physics should read Shelter Island II. One needs a good grasp of quantum electrodynamics and some special mathematics to appreciate the contents fully, but even those without such knowledge will gain an awareness of contemporary developments in theoretical physics. The science-appeal emanating from the book is very strong; I suspect that many physicists will read it initially without understanding everything, and then learn more fundamentals and return to it.

Although the conference was held in 1983 , the book has not dated very much; the various authors remain leading authorities on their subjects and are now, three years later, still developing the ideas discussed in their contributions. Altogether one is grateful to the organizers of the conference and to the editors for bringing together such influential people and for collecting their papers into this excellent book. The publishers might consider issuing a cheaper paperback edition so that its contents would be more widely available, especially to students.

Ya. B. Zeldovich is at the Institute for Physical Problems, USSR Academy of Sciences, Ul. Kosygina 2, Moscow 117973 GSP-1, USSR. 